\title{
Signatures of dark Higgs boson in light fermionic dark matter scenarios
}

\author{
Luc Darmé, ${ }^{a}$ Soumya Rao $^{a}$ and Leszek Roszkowski ${ }^{b, a}$ \\ ${ }^{a}$ National Centre for Nuclear Research, \\ Hoża 69, 00-681 Warsaw, Poland \\ ${ }^{b}$ Astrocent, Nicolaus Copernicus Astronomical Center Polish Academy of Sciences, \\ ul. Bartycka 18, 00-716 Warsaw, Poland \\ E-mail: luc.darme@ncbj.gov.pl, soumya.rao@ncbj.gov.pl, \\ leszek.roszkowski@ncbj.gov.pl
}

ABSTRACT: Thermal dark matter scenarios based on light (sub-GeV) fermions typically require the presence of an extra dark sector containing both a massive dark photon along with a dark Higgs boson. The latter typically generates both the dark photon mass and an additional mass term for the dark sector fermions. This simple setup has both rich phenomenology and bright detection prospects at high-intensity accelerator experiments. We point out that in addition to the well studied pseudo-Dirac regime, this model can achieve the correct relic density in three different scenarios, and examine in details their properties and experimental prospects. We emphasize in particular the effect of the dark Higgs boson on both detection prospects and cosmological bounds.

Keywords: Beyond Standard Model, Dark matter, Fixed target experiments

ArXiv EPRINT: 1807.10314 


\section{Contents}

1 Introduction 1

2 Building a light fermionic DM model 3

2.1 A complete minimal framework 3

2.2 Simplified models and dark Higgs boson $\quad 6$

2.3 Astrophysical and cosmological constraints on the dark Higgs boson 9

3 Dark Higgs boson at accelerator experiments 11

$\begin{array}{lll}3.1 & \text { Dark Higgs boson production and decay } & 11\end{array}$

$\begin{array}{lll}3.2 & \text { Accelerator experiments constraints } & 14\end{array}$

$\begin{array}{llr}4 & \text { Summary and conclusions } & 17\end{array}$

$\begin{array}{ll}\text { A Scan regions and input parameters } & 18\end{array}$

$\begin{array}{lr}\text { B Monte-Carlo setup and numerical simulations } & 19\end{array}$

$\begin{array}{lll}\text { B.1 Production at electron and proton beam dump } & 20\end{array}$

B.2 Decay and detector simulations 21

\section{Introduction}

Thermal Dark matter (DM) candidate with a sub-GeV mass scale has attracted a great deal of attention (for recent reviews see e.g. [1-3]). Much recent effort has gone to explore the nature and properties of $\mathrm{DM}$ of sub-GeV mass interacting through a light mediator of the same scale, hence reproducing WIMP scenarios at the MeV scale. Such scenarios involve the presence of a $\mathrm{MeV}$ scale vector boson charged under an extra $\mathrm{U}(1)_{D}$, usually referred to as dark photon, that acts as the light mediator for dark matter interactions. The kinetic mixing between the dark photon and the Standard Model (SM) photon is then invoked to introduce interactions between the dark sector and SM particles (see $[4,5]$ for reviews). We focus on a model containing a complete, "dark sector" built from a Dirac fermion DM candidate, a dark photon and a dark Higgs boson. Interestingly, bounds from cosmic microwave background (CMB) observations can be avoided by introducing Yukawa couplings between the dark Higgs boson and the DM field which lead to an additional Majorana mass term once the dark Higgs boson acquires its vacuum expectation value.

In this article, we build on our previous work [6] and show that this model exhibits a very rich DM phenomenology. Indeed, we have identified four distinct scenarios which achieve the correct relic density while avoiding the bounds from CMB: (i) an inelastic DM $(i D M)$ regime, (ii) a Majorana DM $(m D M)$ regime, (iii) a secluded regime and (iv) a forbidden regime. The $i D M$ regime is defined as the region of the parameter space with 
pseudo-Dirac DM composed of two Majorana states with a small mass splitting [7]. This scenario has been well studied in the past, and the dark Higgs boson does not play any significant role there (see $[4,5]$ for a review). The $m D M$ regime has larger mass splitting between the two Majorana mass eigenstates, of the order of the DM mass, and markedly different detection prospects in accelerator-based experiments. The secluded regime refers to the region where the DM mass is close to the dark Higgs boson mass such that the annihilation channel into dark Higgs bosons becomes critical in reaching the thermal target. Note that this definition of "secluded" region is different from the one used in previous literature [8-11]. And finally, the forbidden regime is the region of parameter space where the DM mass is close to that of the dark photon but less than that of the dark Higgs boson. Thus, the annihilation channel of DM into dark photon pair or a dark Higgs boson and a dark photon is forbidden kinematically, but nonetheless contribute to relic density due to thermal effects. Finally, note that very similar models but with a dark sector in the GeV range or heavier can also reach the thermal target with an interesting dark matter and LHC phenomenology (see e.g. [12, 13] for recent works).

While direct detection experiments are not typically sensitive to our model due to various suppression mechanisms [5] (since scattering processes are either inelastic or velocitysuppressed), detection prospects in accelerator-based experiments are bright and have been enthusiastically studied in recent years for a variety of contexts and experimental projects (some very recent examples are for instance [14-22]). However, bounds for sub$\mathrm{GeV}$ fermionic DM were mainly obtained in the pseudo-Dirac regime, either by searching for the decay of the long-lived heavy component of the pseudo-Dirac DM, or by focusing instead on the signatures from DM scattering, as advertised in, e.g. [23-28] (a important exception is of course search focusing on the dark photon itself, which remain relatively agnostic about the details of the dark sector, see $[4,5]$ for a review).

Our main point is that the accelerator-based bounds differ significantly in all of the four regimes identified above, especially when the parameter space is restricted to the parameter space reaching the thermal target. In order to illustrate this fact, we focus on two old experiments: LSND [29] that uses a proton beam and a relatively short baseline and the electron beam dump experiment E137 [30], and recast their null results into constraints for each of the four regimes. On top of providing the current experimental status of each regime, this shows that the pseudo-Dirac regime is not the only region of the parameter space which can be significantly constrained by accelerator experiments. Furthermore, we show that when long-lived, the dark Higgs boson can significantly alter the accelerator phenomenology of our model, by either providing sizable new constraints (mainly in the forbidden regime), or on the contrary by reducing dramatically their reach. In particular, while searches for dark Higgs boson had already been advertised long ago in, e.g. [23, 31-33] and considered in our previous work [6], we complement these analyses by including the dark Higgs boson decay into a dark photon and an $e^{+} e^{-}$pair.

The paper is organized as follows. We begin by describing the light fermionic DM model studied here in section 2 . We also describe the different regions of parameter space mentioned above in more detail, and briefly review the cosmological constraints on the dark Higgs boson. In section 3, we present the constraints from beam dump experiments 
like LSND and E137 for each of the scenarios mentioned above. In section 4 we summarize our results and conclude.

\section{Building a light fermionic DM model}

\subsection{A complete minimal framework}

Our goal is to build a model of thermal fermionic DM with mass in the sub-GeV range while relying essentially on simple SM-like building blocks. Arguably one of the most attractive way of doing this is to rely on a vector mediator mechanism, where the portal between the dark sector and the SM is provided by the kinetic mixing between a new, spontaneously-broken, abelian gauge group $\mathrm{U}(1)_{D}$ (under which the $\mathrm{SM}$ is neutral) ${ }^{1}$ and the $\mathrm{SM} \mathrm{U}(1)_{Y}$. In this approach, two main constraints need to be factored in: gauge anomaly cancellation for the dark gauge group, and indirect detection bounds. The former implies that at least two fermionic fields with opposite $\mathrm{U}(1)_{D}$ charge must be added to the theory, and the latter that their mass terms must be at least partially of Majorana type to avoid an $s$-wave annihilation through an off-shell dark photon. Interestingly, both constraints can be straightforwardly satisfied by including a Yukawa coupling between the dark Higgs boson and these new fermionic states.

More precisely, we define a Dirac fermion $\chi=\left(\chi_{L}, \chi_{R}^{\dagger}\right)$ DM with charge 1 under the dark $U(1)$, which will acquire additional Majorana masses from its Yukawa interactions with the dark Higgs boson of $\mathrm{U}(1)_{D}$ charge $q_{S}=+2$. The effective Lagrangian for the dark photon vector $(V)$ and the dark Higgs boson $(S)$ fields in this minimal dark sector model is then given by

$$
\begin{aligned}
& \mathcal{L}_{V}=-\frac{1}{4} F^{\prime \mu \nu} F_{\mu \nu}^{\prime}-\frac{1}{2} \frac{\varepsilon}{\cos \theta_{w}} B_{\mu \nu} F^{\prime \mu \nu} \\
& \mathcal{L}_{S}=\left(D^{\mu} S\right)^{*}\left(D_{\mu} S\right)+\mu_{S}^{2}|S|^{2}-\frac{\lambda_{S}}{2}|S|^{4}-\frac{\lambda_{S H}}{2}|S|^{2}|H|^{2},
\end{aligned}
$$

where $F^{\prime} \mu \nu$ is the corresponding field tensor for $\mathrm{U}(1)_{D}$, and we have introduced a kinetic mixing term parametrized by $\varepsilon$. This term is an invariant of both gauge groups which, even if not present at tree-level in the theory, can be generated by loop corrections of heavy vector-like fermion charged under both $\mathrm{U}(1) \mathrm{s}$ [35]. In practice, one then removes the kinetic mixing term by transforming the gauge fields so as to obtain diagonal kinetic terms and ensure that the photon remains massless. In the resulting theory, the $\mathrm{U}(1)_{Y}$-charged Standard Model fields acquire an $\varepsilon$-suppressed $\mathrm{U}(1)_{D}$-charge, while the dark sector remains electrically neutral (see, e.g. [36]). Furthermore, we will fix the value of the gauge-preserving quartic coupling mixing the dark Higgs boson with the SM Higgs to zero, since, as was shown in [6], its effects are negligible for values compatible with a natural splitting between dark sector and the SM (see also [37] where this coupling is generated directly by supersymmetry, as is shown to have small values). The DM field is then introduced through the

\footnotetext{
${ }^{1}$ Note that building models where part of the SM fields are charged under the new dark gauge group is possible but non-trivial, see e.g. [34].
} 
Lagrangian

$$
\mathcal{L}^{\mathrm{DM}}=\bar{\chi}\left(i \not D-m_{\chi}\right) \chi+\left(y_{L} S \bar{\chi}^{c} P_{L} \chi+y_{R} S \bar{\chi}^{c} P_{R} \chi+\text { h.c. }\right)
$$

After the dark Higgs boson acquires a Vacuum Expectation Value (VEV), the mass of the dark Higgs boson and of the dark photon become correlated and are given by

$$
\begin{aligned}
& M_{S}=\sqrt{2 \lambda_{S}} v_{S} \\
& M_{V}=g_{V} q_{S} v_{S}=\left(\frac{q_{S} g_{V}}{\sqrt{2 \lambda_{S}}}\right) M_{S} .
\end{aligned}
$$

In term of Weyl fermions, the interaction and mass terms for the DM fields are

$$
\begin{aligned}
\mathcal{L}^{\mathrm{DM}} \supset & \left(\frac{1}{2}\left(\chi_{L}, \chi_{R}\right) M_{\chi}\left(\begin{array}{c}
\chi_{L} \\
\chi_{R}
\end{array}\right)+\frac{y_{L}}{\sqrt{2}} S \chi_{L} \chi_{L}+\frac{y_{R}}{\sqrt{2}} S \chi_{R}^{\dagger} \chi_{R}^{\dagger}+\text { h.c. }\right) \\
& +\chi_{R}^{\dagger} \bar{\sigma}^{\mu} V_{\mu} \chi_{R}-\chi_{L}^{\dagger} \bar{\sigma}^{\mu} V_{\mu} \chi_{L},
\end{aligned}
$$

where the DM mass matrix is

$$
M_{\chi}=\left(\begin{array}{cc}
\sqrt{2} v_{S} y_{L} & m_{\chi} \\
m_{\chi} & \sqrt{2} v_{S} y_{R}
\end{array}\right) .
$$

The lightest eigenstate has typically a negative mass and the splitting between the two mass eigenstates $\chi_{1}$ and $\chi_{2}$ is

$$
\Delta_{\chi} \equiv M_{\chi_{1}}+M_{\chi_{2}}=\sqrt{2} \frac{M_{V}}{q_{s} g_{V}}\left(y_{R}+y_{L}\right)
$$

As usual in this case, there are typically two possible conventions, one can either keep the negative mass but ensure that the rotation matrix in the DM sector $Z^{X}$ is real, or else ensure that both eigenstates have positive masses, at the expense of introducing an imaginary rotation matrix. Following [7], most authors considering the pseudo-Dirac limit have used the latter choice as this leads to a very simple form for the gauge interaction between mass eigenstates $\chi_{1}$ and $\chi_{2}$. We will make throughout this paper the opposite choice to have potentially a negative mass for $\chi_{1}$ but real rotation matrices as this is easier to implement numerically while not in the pseudo-Dirac limit. In the following, we will also denote by $\chi_{1}$ and $\chi_{2}$ the Majorana fermions corresponding to the previously defined Weyl fermion states for notational simplicity. We also use the notation $M_{\chi}$ (e.g in plots) to refer to the absolute value of the DM mass $M_{\chi}=\left|M_{\chi_{1}}\right|$ and when $M_{\chi_{1}}>0$, we define $\Delta_{\chi} \equiv M_{\chi_{2}}-M_{\chi_{1}}$.

Defining the effective gauge interaction between $\chi_{1}$ and $\chi_{2}$ by $g_{D, 12}$, we have

$$
g_{D, 12}=g_{V} \frac{1}{\sqrt{1+\frac{M_{V}^{2}\left(y_{L}-y_{R}\right)^{2}}{2 g_{V}^{2} m_{\chi}^{2}}}},
$$


which reduces to the dark gauge coupling $g_{V}$ when the Dirac mass dominates, as expected. On the other hand, the DM candidate $\chi_{1}$ has an effective gauge coupling given by

$$
g_{D, 11}=g_{V} \frac{M_{V}\left(y_{L}-y_{R}\right)}{\sqrt{2 g_{V}^{2} m_{\chi}^{2}+M_{V}^{2}\left(y_{L}-y_{R}\right)^{2}}},
$$

which vanishes in the limit where $y_{L}=y_{R}$ and is very small for $y_{L}, y_{R} \ll 1$ or $y_{L} \sim y_{R}$.

A key element of this model is the fact that there are only two independent mass scales: the fermion Dirac mass and the dark Higgs boson VEV whose interplay will determine most of the phenomenology of the model. As we will see later, the DM mass is typically restricted by CMB bounds and relic density evaluation to be smaller than the dark photon one: $M_{\chi}<M_{V}$. Freedom in the spectrum of the model is therefore mainly determined by the position of the dark Higgs boson mass and the four regimes we will identify below largely depends on it.

Analytically, we find that the dark Higgs boson is lighter than the dark photon when

$$
\sqrt{2 \lambda_{S}}<q_{S} g_{V}
$$

A similarly simple expression can be found to assess whether or not the dark Higgs boson is lighter than the splitting between the two fermion states $\chi_{1}$ and $\chi_{2}$. When $M_{\chi_{1}}<0$, one has $M_{S}<\Delta_{\chi}$ when

$$
\lambda_{S}<4\left(y_{R}+y_{L}\right)^{2}
$$

While the Higgs quartic and the dark gauge coupling are a priori free parameters of our model, we can place an upper bound by requiring them to remain perturbative at least up to the $\mathrm{TeV}$ scale. ${ }^{2}$ Considering the gauge coupling alone leads to the usual result $\alpha_{D} \equiv g_{V}^{2} /(4 \pi) \lesssim 0.5$. On the other hand, and especially in the pseudo-Dirac regime where $y_{L}, y_{R} \ll 1$, the Higgs quartic beta function is given by

$$
\beta_{\lambda_{S}}=96 g_{V}^{2}+20 \lambda_{S}^{2}-48 g_{V}^{2} \lambda_{S},
$$

and is positive. In the naive assumption of constant gauge coupling we get $\lambda_{S}^{1 \mathrm{TeV}}=$ $\lambda_{S}^{100 \mathrm{MeV}} \exp \left(8\left(\frac{\alpha_{D}}{0.1}\right)^{2}\right)$. In practice, perturbativity of the dark Higgs quartic hence typically limits $\alpha_{D} \lesssim 0.1-0.15$ depending on the size of the negative contributions from the Yukawa coupling $y_{L}, y_{R}$ and of the initial value of $\lambda_{S}$. In the rest of this paper, we make sure that our parameter space satisfies perturbativity by evaluating the spectrum obtained by using SPheno $[38,39]$ code created by SARAH [40-42]. In particular, we run the initial couplings up to the $\mathrm{TeV}$ scale to check that they remain perturbative, then evaluate all masses at the sub-GeV scale we are interested at tree-level.

\footnotetext{
${ }^{2}$ Assumed to be the typical mass scale of the SM-charged vector-like fermions creating the kinetic mixing parameter.
} 


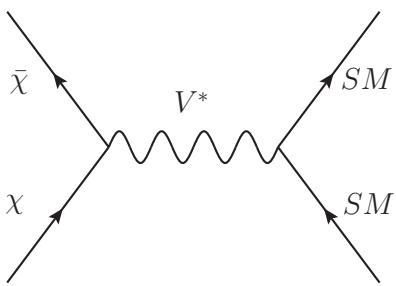

(a)

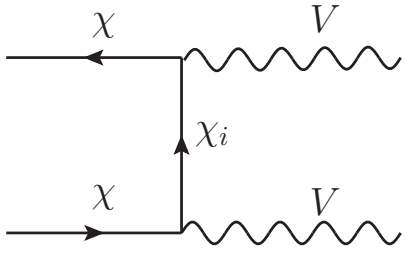

(b)

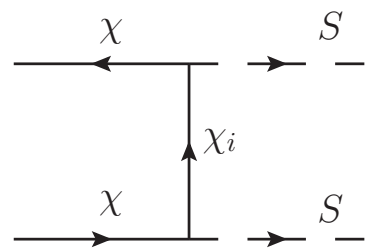

(c)

Figure 1. Dominant DM annihilation channels (note that $\chi$ can be either $\chi_{1}$ or $\chi_{2}$ depending whether or not coannihilation is relevant). The processes are (a) S-channel annihilation through an off-shell dark photon, (b) Thermally-induced forbidden annihilation into dark photons, (c) Secluded annihilation into two dark Higgs bosons.

\subsection{Simplified models and dark Higgs boson}

Our numerical results presented in the rest of this section are based on a scan of the parameter space of our model (see appendix A for the input parameters and the chosen range) to identify all relevant DM regions. We used MultiNest [43] to direct the scanning process toward relic density values compatible with the result from the Planck Collaboration [44] $\Omega h^{2}=0.1188 \pm 0.0010$. The code BayesFITS is used to interface all the public codes used, including a slightly modified version of MicrOMEGAs v.4.3.5 [45], SPheno, and a heavily modified version of the code BdNMC from [26].

In this simple model, the correct relic density of DM is typically obtained through three main diagrams shown in figure 1. Crucially, each of them suppresses the annihilation of DM at the time of CMB recombination, as required by the stringent bounds on the annihilation process [46]. In particular, the $s$-channel annihilation corresponding to the diagram of figure 1(a) is either population-suppressed in the pseudo-Dirac case $\chi_{1} \chi_{2} \rightarrow V^{*} \rightarrow e^{+} e^{-}$ since all $\chi_{2}$ states have decayed at recombination time, or it is velocity-suppressed in the Majorana DM case since $\chi_{1} \chi_{1} \rightarrow V^{*} \rightarrow e^{+} e^{-}$is a $p$-wave process. Next, while the $t$-channel annihilation of figure 1(b) into dark photons is an $s$-wave process, it has to proceed through thermal effects in the kinematically forbidden regime $M_{\chi}<M_{V}$ to give the correct relic density. Finally, the $t$-channel annihilation into dark Higgs bosons of figure 1(c) is also of $p$-wave type and thus velocity-suppressed. Note that the annihilation into $S V$ can also lead to the correct relic density in a similar fashion as the $V V$ channel, albeit in a smaller region of the parameter space (we include this region into the larger "forbidden regime" one in the following).

Based on these processes, we have identified four typical scenarios where one can obtain the correct DM relic density while avoiding CMB bounds: the inelastic DM regime (iDM), the Majorana DM regime $(\mathrm{mDM})$, the secluded regime and the forbidden regime. These four scenarios are represented in the $M_{S} / M_{\chi}$ plane in figure 2. As was underlined in [6], when the dark Higgs boson is long-lived (typically $M_{S}<2 M_{\chi}$ and $M_{S}<M_{V}$ ), the relic density must be obtained by considering a two-component DM-like scenario to obtain simultaneously the correct relic density and the dark Higgs boson metastable density. An 


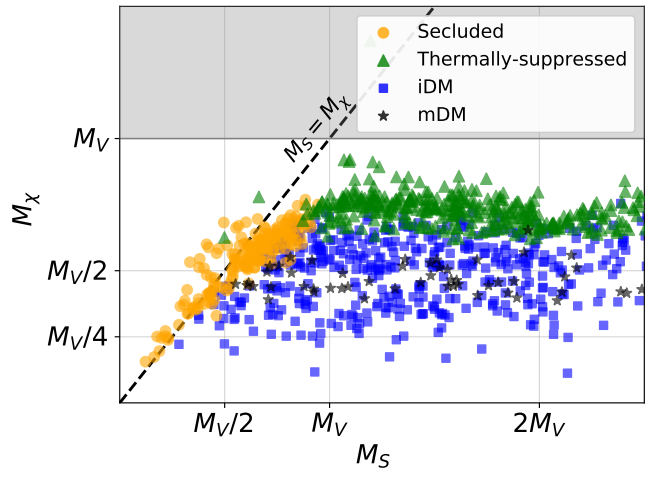

(a)

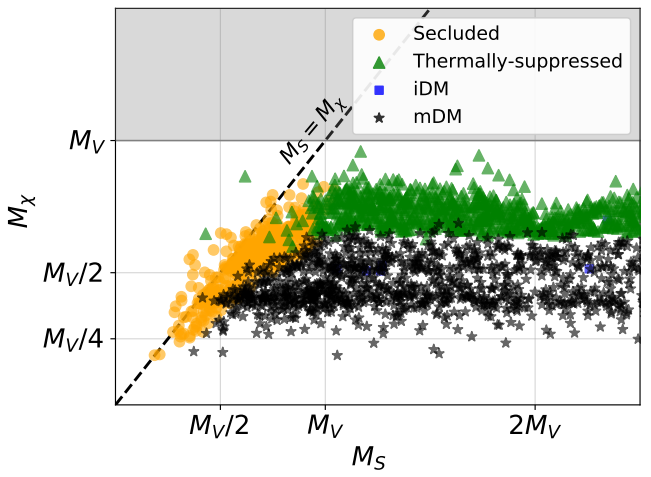

(b)

Figure 2. Data points satisfying BBN bounds and the relic density constraint from the numerical scan described in the main text, represented in the $\left(M_{S},\left|M_{\chi}\right|\right)$ plane with masses in unit of $M_{V}$ for (a) $\Delta_{\chi}<0.5 M_{\chi}$ and (b) $\Delta_{\chi}>0.5 M_{\chi}$. We have classified them in four regimes: inelastic DM (blue squares), Majorana DM (black stars), secluded regime (orange circles), and forbidden regime (green triangles).

important comment to make is that these four regimes corresponds to a selected range of parameter space of the proposed model, as shown in table 2. In particular, we do not probe very small values of our parameters as these are typically considered when additional symmetries are assumed and lead to a different phenomenology: for instance, a dark charge conjugation symmetry to set the kinetic mixing term to zero (as done recently in, e.g. [47]), or strongly suppressed Yukawa couplings due to an approximate $\chi$-number global symmetry which could lead to kinematically forbidding the $\chi_{2} \rightarrow \chi_{1} e^{+} e^{-}$decays.

Let us review the properties of each scenario in turn:

- Inelastic DM regime (iDM): this scenario has been the most studied one in the literature. It roughly corresponds to the mass spectrum $M_{\chi}<M_{S}, M_{V}$ with $M_{\chi_{1}}<0$ in our convention and $\Delta_{\chi}<0.5 M_{\chi}$ as can be seen in figure 2. In this regime the role of the dark Higgs boson is typically neglected and an approximate number symmetry is introduced so that $y_{R}, y_{L} \ll 1$. Under this assumption, DM is of the pseudoDirac type and the mechanism to obtain the correct relic density is a coannihilation through an off-shell dark photon. This scenario has the advantage of achieving the correct relic density for a wide range of parameters and leading to strong signatures in accelerator experiments. A typical spectrum in this scenario is

$$
M_{V}: M_{\chi}: \Delta_{\chi}=3: 1: 0.1 \text { and } M_{S}>M_{\chi} \text {, }
$$

with couplings of typical order $g_{11} \sim 0, g_{12} \sim g_{V}$ and $y_{\mathrm{DM}} \ll 1$. Note that a priori the dark Higgs boson mass is a free parameter in this scenario, as long as $M_{\chi}<M_{S}$. We show in figure 3 the transition between the two regimes: $M_{\chi}<M_{S}$ and $M_{\chi}>M_{S}$ (secluded regime, described below). An interesting observation is that one should strictly speaking also impose $M_{\chi_{2}}<M_{S}$ since for $M_{\chi}<M_{S}<M_{\chi_{2}}$ the dark Higgs 


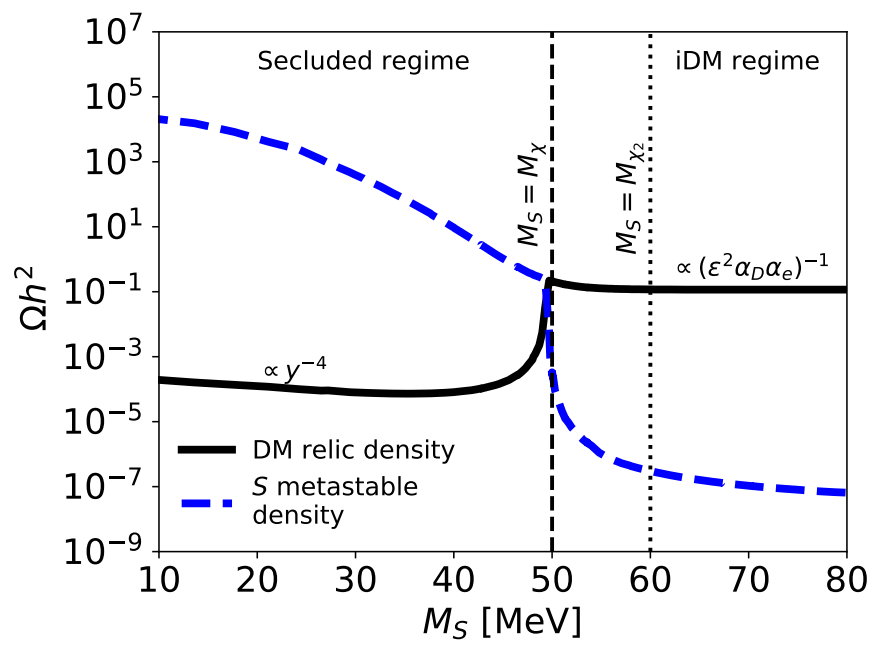

Figure 3. Dark matter relic density $\Omega h^{2}$ and dark Higgs boson metastable abundance $\Omega h_{S}^{2}$ (normalized to the relic density it would have had today if it had been stable) as function of the dark Higgs boson mass. We have chosen $M_{\chi}=50 \mathrm{MeV}, M_{\chi_{2}}=60 \mathrm{MeV}$, and the rest of the couplings so that the correct relic density is obtained in the iDM regime when $M_{S}>M_{\chi_{2}}$.

boson already modifies significantly the relic density by depleting the abundance of the heavy state $\chi_{2}$ and hence reducing the efficiency of the coannihilation mechanism. For larger values of $\Delta_{\chi}$ (above $0.1 M_{\chi}$ ), this scenario is in fact already quite constrained by accelerator-based experiments if the $\chi_{1}$ is assumed to make for the whole DM relic density, as we will see in the next sections.

- Majorana regime ( $m D M)$ : when the constraints on the Yukawa couplings are relaxed and $y_{R}, y_{L} \sim 1$, the Majorana mass can be of the same order or larger as the Dirac mass, leading to a Majorana DM candidate with sizable coupling both to the dark Higgs boson and to the dark photon. The mass spectrum in this regime is typically $M_{\chi}<M_{S}, M_{V}$, with $M_{\chi_{1}}>0$ in our convention and $\Delta_{\chi} \sim M_{\chi}$ as can be seen in figure 2. This scenario shares many similarities with the iDM one but can lead to different limits at accelerator-based experiments. A typical mass ratio in this scenario is

$$
M_{V}: M_{\chi}: \Delta_{\chi}=3: 1: 1
$$

where $M_{\chi}$ is taken positive. ${ }^{3}$ Like in the previous case, as long as $M_{\chi}<M_{S} \ll$ $M_{V}$ the dark Higgs boson mass usually does not play a role in the determination of the relic density. In addition, and similarly to the iDM regime, the parameter space where $\chi_{1}$ is the dominant component of DM is already severely constrained by accelerator searches.

- Secluded regime: when the dark Higgs boson mass becomes of the same order or lighter than the DM particle $M_{S} \sim M_{\chi}$, the relic density is mainly fixed by $t$-channel

\footnotetext{
${ }^{3}$ As described in the appendix $\mathrm{A}$, we then have to fix $y_{L}$ and $y_{R}$ so as to be consistent with this mass ratio.
} 
annihilation into a pair of dark Higgs bosons (figure 1(c)), corresponding to the left-hand side of figure 3. Since the annihilation proceeds through an unsuppressed $t$-channel process, low values $y_{R}, y_{L} \ll 1$ are typically preferred to obtain the correct relic density, which in turn leads to small splitting between $\chi_{1}$ and $\chi_{2}$. Notice that our "secluded' regime differs slightly from the one of [5] since we consider annihilation into the scalar dark Higgs boson and not into the dark photon. Accelerator signatures of this regime are therefore similar to the iDM regime, but the relic density is independent of the kinetic mixing, leading to a larger range of accessible parameter space. A typical mass ratio for this scenario is

$$
M_{S}: M_{\chi}: M_{V}: \Delta_{\chi}=5 / 6: 1: 5 / 2: 0.1 .
$$

Note that in general this scenario can simultaneously be probed by accelerator-based experiments and BBN/CMB observables since the dark Higgs boson can have a very large metastable density after freeze-out, as shown in figure 3 . In the regime where $M_{S} \gtrsim M_{\chi}$ and $M_{S} \lesssim M_{V}$ the DM relic density is fixed by a mechanism sharing similarities with the sterile coannihilation presented in [48]. Overall, the relic density of the whole dark sector (including dark Higgs boson and DM) depends on the thermally-suppressed process $S S \rightarrow V V$ and hence on the dark photon mass. ${ }^{4}$

- Forbidden regime: when the DM mass moves closer to the dark photon one, the $t$-channel annihilation into dark photons of figure 1(b) becomes dominant due to thermal effects, this is the so-called "forbidden regime". In this setup, our model resembled the one of [49], albeit without a pure Dirac DM scenario. A typical mass ratio in this scenario is

$$
M_{S}: M_{\chi}: M_{V}=6 / 5: 4 / 5: 1 \text {. }
$$

Similarly to the previous case, the relic density is then independent of the kinetic mixing parameter. On the other hand, the dark photon decays visibly leading to many more signatures at accelerator experiments. Both the heavy state $\chi_{2}$ and the dark Higgs boson have little impact on the relic density. An interesting exception occurs in the limit $y_{R} \simeq y_{L}$, for which the coupling between the Majorana DM and the gauge boson approximately vanishes. In this limit, an annihilation process of the form $\chi \chi \rightarrow V V$ can nonetheless proceed through an $s$-channel dark Higgs boson exchange, albeit at a slightly suppressed rate, which in turn leads to DM masses closer to the dark photon one. Notice that for really small gauge coupling $g_{V} \sim 0.01$, the relic t-channel annihilation is suppressed enough to lead to the correct relic density for $M_{\chi} \sim M_{V}$, but is excluded by CMB bounds.

\subsection{Astrophysical and cosmological constraints on the dark Higgs boson}

While the dark Higgs boson is an important ingredient of the scenarios described above, its long lifetime when $M_{S}<M_{V}$ and $M_{S}<2 M_{\chi}$ can also lead to various cosmological constraints.

\footnotetext{
${ }^{4}$ While typical metastable dark Higgs boson abundance our of reach of BBN bounds, they are nonetheless constrained by CMB bounds on decaying DM if the dark Higgs boson is extremely long-lived.
} 
BBN and other cosmological bounds. The effect of long-lived scalars like the dark Higgs boson on BBN was studied in [50] and it was seen that stringent constraints on $\varepsilon$ can be placed, restricting the lifetime of a scalar like dark Higgs boson to $<0.1 \mathrm{~s}$. However, it was shown in [6] that such strong constraints can be avoided with the addition of a DM candidate as considered here. In particular, constraints from early time energy injection $(t \lesssim 10 \mathrm{~s}$ ) can be avoided through the efficient annihilation channels (for e.g. $S S \rightarrow \chi \chi$ ) that are possible in this model, while late time energy injection $(t \gtrsim 100 \mathrm{~s})$ constraints are mitigated by kinematically restricting the decay modes of dark Higgs boson to leptonic products only.

For longer lifetimes (corresponding to $\varepsilon \lesssim 10^{-6}$ ), bounds from CMB deformation (see, e.g. [51]) due to dark Higgs boson decays during the recombination era becomes relevant and can probe a dark Higgs boson metastable density up to $\Omega h_{S}^{2} \sim 10^{-12}$.

Furthermore, when the DM mass is around $5 \mathrm{MeV}$ and below, CMB bounds on the effective number of neutrinos arise [52], with some model dependency on the precise value of the limit. In the same mass range and for strong gauge coupling $\alpha_{D} \gtrsim 0.5$, constraints from self-interaction of the DM may also become relevant [5].

Constraints from supernova 1987A. Constraints on light dark sector particles in the MeV range from supernova 1987A (SN1987A) [53-56] have been studied in several articles [31, 57-65]. We briefly comment about the relevance of the constraints obtained in [65] for an inelastic DM model that is similar to the model studied here.

A core-collapsed supernova like SN1987A gives rise to a hot neutron star or a protoneutron star environment. The creation of new particles that can interact with SM particles and their subsequent escape can lead to cooling of the interior of the proto-neutron star. The core of the proto-neutron star is around $T \sim 30 \mathrm{MeV}$ and a significant loss of energy in the form of dark sector particles in the $\mathrm{MeV}$ range can result in the supernova cooling at a faster rate than expected. In previous studies, the dark photon production inside the supernova core through bremsstrahlung process has been used to constrain the kinetic mixing parameter $\varepsilon$. A recent update of this constraint includes a DM candidate in addition to the dark photon [65]. In the context of the model studied here, the dark sector includes a further addition of the dark Higgs boson.

For SN1987A constraints on DM in our model, the inelastic DM case when $\Delta=0$ is the relevant case considered in [65]. Note that the constraints for $\Delta>0$ are not directly applicable here, because of the model restrictions used in [65]. In particular, the presence of elastic scattering $\chi_{1} p \rightarrow \chi_{1} p$ is neglected, unlike the case for our model where it becomes significant when the splitting $\Delta_{\chi}$ increases (see eq. (2.10)).

In principle the effect of this constraint on dark Higgs boson should be mitigated by two reasons: the production of dark Higgs boson being suppressed compared to dark photon and DM, and the ambiguity of the distribution of DM and dark photon inside the supernova particularly for the large values of $\varepsilon$ and other relevant couplings considered here, for which the dark Higgs boson can scatter off dark photon and/or DM at a significantly large rate. Obtaining a proper lower limit on the parameter space will require a calculation involving full simulation of the dark sector population inside the supernova. 


\section{Dark Higgs boson at accelerator experiments}

We present in this section the bounds on the four scenarios presented above that arise from accelerator-based experiments. The fact that the dark sector in our model not only contains a dark photon and a DM particle, but also a dark Higgs boson and a heavy dark sector state $\chi_{2}$ will significantly improve the experimental prospects.

Most generic searches typically focus on a dark photon and either assume that it decays invisibly and search for missing-energy signatures [66, 67] - the strongest bounds using this strategy are currently from BaBar [68] and NA64 [69]. Alternatively, one can also try to observe its decay as bumps in a dilepton invariant spectrum such as in NA-48/2 [70], BaBar [71] and LHCb [72]. If the dark photon decays visibly but with a longer lifetime then a range of long-baseline experiments (see [4] for a review) further probe the range $\varepsilon \lesssim 10^{-4}-10^{-5}$.

One can also search for scattering of DM particles produced through on-shell dark photon decay in various beam dump experiment. This has been an active field of research for a decade [23-27, 73, 74], and we have included the case of scattering in the LSND experiment as an example of the current sensitivity. ${ }^{5}$

In this work, while including the previous bounds, we focus instead on stronger but more model-dependent bounds arising from the decay of either the heavy dark sector state $\chi_{2}$ or of the dark Higgs boson. In particular, we complement our previous work [6, 75] in several directions by including more production channels for the dark Higgs boson and by considering also its decay channel through an off-shell dark photon, which becomes dominant when $M_{S}>M_{V}$. Furthermore, we have also included the bounds from the decay of the long-lived heavy dark sector state $\chi_{2} \rightarrow \chi_{1} e^{+} e^{-}$, studied in [37] in a supersymmetric context and later by [73] in a setup similar to our iDM regime. Being obtained with a completely different code, our results can be considered as an independent cross-check for the iDM regime.

\subsection{Dark Higgs boson production and decay}

In accelerator-based experiment, the dark Higgs boson is mainly produced through either the chain decay of some heavy dark sector states (for example $\chi_{2}$, as shown in figure 4(a)), when this is kinematically allowed, or through dark Higgstrahlung as shown in figure 4(b). In general, the former has higher rates but at the expense of a stronger model dependence. Both processes can happen either in proton beam dump experiments, for instance from meson decay, or in electron beam-dump experiment through bremsstrahlung production of dark photon (dark bremsstrahlung). Note that while bremsstrahlung is also present in proton beam-dump experiment, it is typically subdominant compared to meson decay when $M_{V} \lesssim 0.5 \mathrm{GeV}$ (see, e.g. [20,26]) and was found to be altogether negligible in LSND due to the very low energy of the initial beam [73].

\footnotetext{
${ }^{5}$ While scattering bounds also exist for E137 and recently for miniBooNE, they have typically a reach relatively similar to LSND [74]. We therefore focus on the LSND case in the following.
} 
For the dark bremsstrahlung production (in our case relevant for the electron beam dump experiment E137) we have used the public code Madgraph [76]. Some details about numerical setup and the target form factors used in the calculation are given in appendix B.

For the meson decay cases, we use a modified version of the code BdNMC [26] where we have directly included the expression for the various differential branching ratios relevant to both the dark Higgs boson and heavy dark sector states production. We refer to [6] for the details of the dark Higgstrahlung process in the case of proton beam dumps. In this work, we will focus on the remaining expressions which are needed for the dark sector state production.

In order to treat the chain decay case in proton beam dump, we have recalculated the production rates for DM in meson decays through an on-shell or off-shell dark photon including the proper rotation matrices for the dark sector states $\chi_{1}$ and $\chi_{2}$. This allows one to smoothly interpolate between the various regimes described above (in particular, we recover the standard results from ref. [73] for the iDM regime). As in [6], we are interested in the differential decay rate $d \mathrm{BR}_{\pi^{0} \rightarrow \gamma \chi_{i} \chi_{j}} / d s d \theta^{V}$, where $s$ denotes the four-momentum squared of $V^{*}$, and where, calling $\vec{X}_{V}$ the direction of the mediating dark photon $V^{*}$ in the rest frame of the initial $\pi^{0},{ }^{6} \theta^{V}$ denotes the angle between $\chi_{i}$ and $\vec{X}_{V}$ in the $V^{*}$ rest frame. A key parameter is the coupling $g_{i j}$ between $\chi_{i} \chi_{j}$ and $V$, which is already described analytically in eq. (2.9) and eq. (2.10). In term of the rotation matrix $Z^{X}$ relating the DM gauge eigenstates to the mass eigenstates, it is given by

$$
g_{i j}=g_{V}\left(Z_{i 1}^{X} Z_{j 1}^{X}-Z_{i 2}^{X} Z_{j 2}^{X}\right),
$$

and the vertex includes a $\gamma^{\mu} \gamma^{5}$ factor in our conventions (real mixing matrices but potentially negative mass for $\chi_{1}$ ). We obtain

$$
\begin{aligned}
\frac{d^{2} \mathrm{BR}_{\pi^{0} \rightarrow \gamma \chi_{i} \chi_{j}}=}{d s d \theta^{V}}= & \mathcal{S} \times \mathrm{BR}_{\pi^{0} \rightarrow \gamma \gamma} \times g_{i j}^{2} \times \\
& \frac{\varepsilon^{2} \alpha_{D}}{4 \pi} s\left(1-\frac{s}{m_{\pi^{0}}^{2}}\right)^{3} \times \frac{\sqrt{\lambda}\left(2 s\left[s-\left(M_{i}+M_{j}\right)^{2}\right]-\lambda \sin ^{2} \theta^{V}\right)}{\left(s-M_{V}^{2}\right)^{2}+M_{V}^{2} \Gamma_{V}^{2}} \sin \theta^{V},
\end{aligned}
$$

where $\mathcal{S}$ is a symmetry factor equal to $1 / 2$ if $i=j$ and 1 otherwise, $\Gamma_{V}$ is the width of the dark photon and the triangular function $\lambda$ is defined as

$$
\lambda \equiv\left(1-\frac{\left(M_{i}+M_{j}\right)^{2}}{s}\right)\left(1-\frac{\left(M_{i}-M_{j}\right)^{2}}{s}\right) .
$$

As usual, this straightforwardly applies to the case of the $\eta$ meson by replacing $m_{\pi^{0}}$ by $m_{\eta}$ and $\mathrm{BR}_{\pi^{0} \rightarrow \gamma \gamma}$ by $\mathrm{BR}_{\eta \rightarrow \gamma \gamma}=0.394$. The subsequent decay of the heavy dark sector state $\chi_{2}=\chi_{1} S$ is then considered to occur instantaneously and with a branching ratio of one if it is kinematically accessible.

\footnotetext{
${ }^{6}$ Equivalently, calling $\overrightarrow{p_{i}}$ and $\overrightarrow{p_{j}}$ the three-momenta of $\chi_{i}$ and $\chi_{j}$ in the rest frame of the initial $\pi^{0}, \vec{X}_{V}$ is the direction of $\overrightarrow{p_{i}}+\overrightarrow{p_{j}}$, which is of course not modified while boosting to the rest frame of the $V^{*}$.
} 


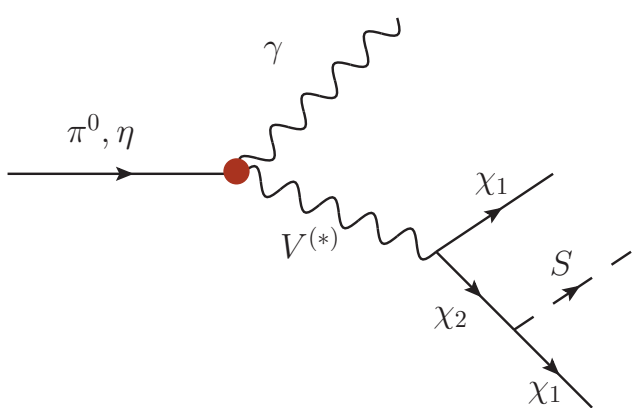

(a)

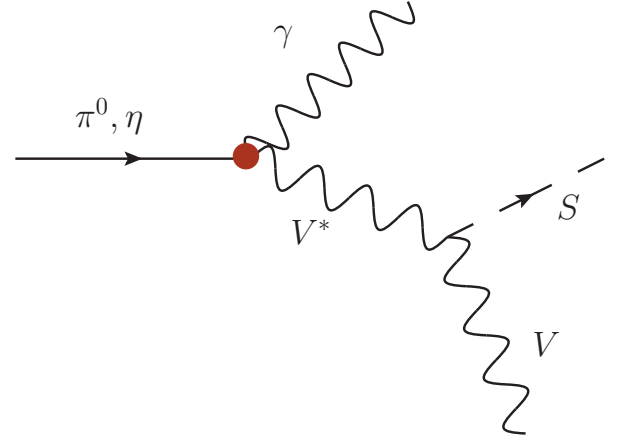

(b)

Figure 4. Dark Higgs boson production in meson decay through (a) chain decay of a heavy dark sector state and (b) dark Higgstrahlung.

We are interested in signatures corresponding to the decay product of the dark Higgs boson. In the mass range we consider and if $M_{S}<2 M_{\chi}, M_{S}<M_{V}$, the dominant decay channel is a loop-induced decay into an electron-positron pair, leading to an extremely long lifetime $[6,10]$,

$$
\tau_{S} \propto 10 \mathrm{~s} \times\left(\frac{\alpha_{\mathrm{em}}}{q_{S}^{2} \alpha_{D}}\right)\left(\frac{10^{-3}}{\varepsilon}\right)^{4}\left(\frac{50 \mathrm{MeV}}{M_{S}}\right)\left(\frac{M_{V}}{100 \mathrm{MeV}}\right)^{2} .
$$

On the other hand if $M_{V}+2 m_{e}<M_{S}<2 M_{\chi}$, the decay channel $S \rightarrow V e^{+} e^{-}$becomes kinematically accessible. Interestingly, for relatively small splitting, the decay width takes a simple form

$$
\Gamma_{S}=\frac{16 q_{S}^{2} \alpha_{D} \alpha_{\mathrm{em}} \varepsilon^{2}}{15 \pi} \times \frac{\left(M_{S}-M_{V}\right)^{5}}{M_{V}^{4}}
$$

which can be parametrically expressed as

$$
c \tau_{S} \propto 20 \mathrm{~m} \times\left(\frac{0.1}{\alpha_{D}}\right)\left(\frac{10^{-4}}{\varepsilon}\right)^{2}\left(\frac{0.1 M_{V}}{M_{S}-M_{V}}\right)^{5}\left(\frac{100 \mathrm{MeV}}{M_{V}}\right) .
$$

As it was already noticed in [33] in a related context, the dark Higgs boson lifetime is now significantly shorter and scales only as $\varepsilon^{2}$, similarly to the heavy dark sector decay considered in [73]. This allows one to probe extremely small values of $\varepsilon$ since a sizable fraction of the dark Higgs bosons produced in accelerator-based experiments will decay in the detector. ${ }^{7}$

\footnotetext{
${ }^{7}$ It is interesting to compare this result with the expression for the lifetime of the long-lived heavy dark sector state $\chi_{2}$ which can be easily derived from the results of [73],

$$
c \tau_{\chi_{2}} \propto 100 \mathrm{~m} \times\left(\frac{0.1}{\alpha_{D}}\right)\left(\frac{10^{-3}}{\varepsilon}\right)^{2}\left(\frac{0.2 M_{\chi}}{\Delta_{\chi}}\right)^{5}\left(\frac{25 \mathrm{MeV}}{M_{\chi}}\right)^{5}\left(\frac{M_{V}}{100 \mathrm{MeV}}\right)^{4} .
$$




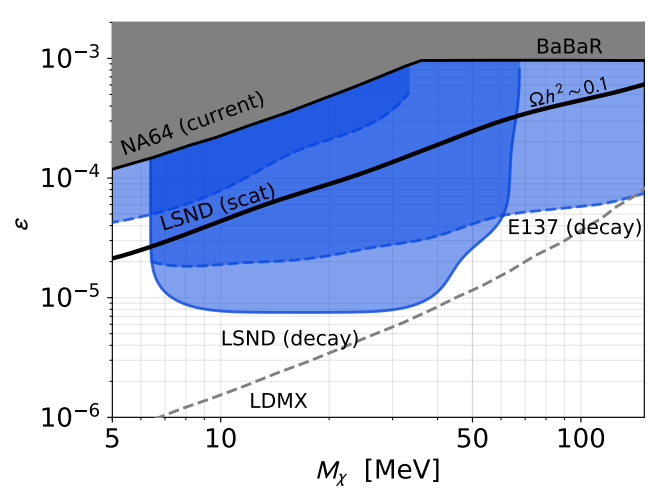

(a)

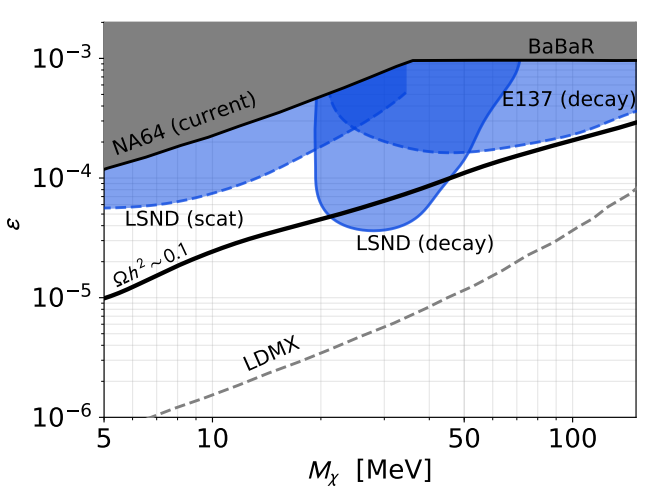

(b)

Figure 5. Bounds on the inelastic DM regime from the E137 [30], LSND [77] arising from DM scattering and $\chi_{2}$ decay (blue shaded regions) and missing energy searches (grey regions and grey dashed line for the LDMX projection from [5]). The thick black lines correspond to points featuring the correct relic density of DM. We fixed $\alpha_{D}=0.1, y_{\mathrm{DM}}=0.01$ and varied $M_{\chi}$ and $\varepsilon$ using the mass ratios $M_{S}: M_{\chi}: M_{V}=2: 1: 3$ for (a) $\Delta_{\chi}=0.15 M_{\chi}$ and (b) $\Delta_{\chi}=0.05 M_{\chi}$.

\subsection{Accelerator experiments constraints}

In the recent years, there has been a surge in interest in examining the potential reach of upcoming neutrino-related experiments in probing light DM sectors. In this section, we rather take the approach of considering the currently applicable bounds from the existing experiments LSND [77] and E137 [30] to explore the current limits on the four scenarios described above. In particular, we want to point out in which case the presence of the dark Higgs boson affects strongly the constraints and phenomenology of the model. We want to study in detail the signatures from the secluded, forbidden and Majorana DM regime which have not been studied in details so far. We refer to the appendix for more details on the Monte-Carlo simulation used to get the expected number of events.

Inelastic DM regime (iDM). This regime has been already thoroughly analyzed in the context of both existing experiments and for several prospective ones. Furthermore, as shown in [6], in this case the dark Higgs boson is extremely long-lived, so that the bounds from dark Higgs boson-related processes are not relevant. On the other hand, the decay of long-lived heavy dark sector states give strong signatures in many existing and upcoming fixed-target and accelerator-based experiments. In order to cross-check our numerical tools, we have reproduced in figure 5 the current constraints which arise from the LSND and E137 experiments and found very good agreement with the existing literature.

Majorana regime $(\mathbf{m D M})$. When the mass splitting between $\chi_{1}$ and $\chi_{2}$ increases, the heavy state can decay before reaching the detector. This typically happens for values of the kinetic mixing parameter below the missing energy search limit when $\Delta_{\chi} \gtrsim M_{\chi}$ for LSND and even lower values in E137 as can be seen in figure 6. Consequently, the limits from, e.g., LSND present now an upper bound for these types of process. We illustrate this 


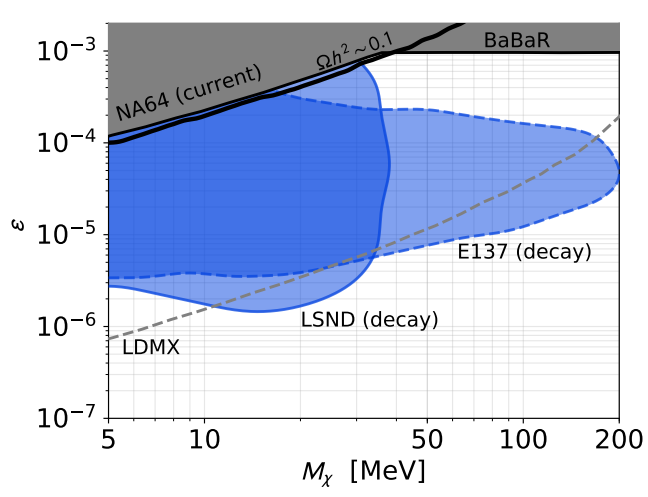

(a)

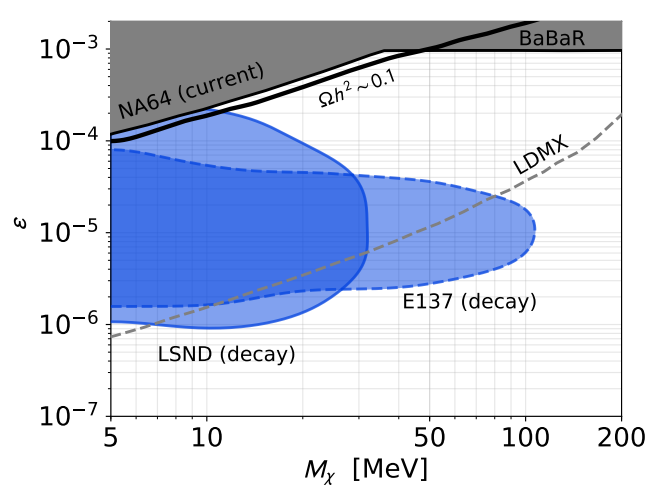

(b)

Figure 6. Bounds on the Majorana DM regime from the E137 [30], LSND [77] arising from DM scattering and $\chi_{2}$ decay (blue shaded regions) and missing energy searches (grey regions and grey dashed line for the LDMX projection from [5]). The thick black lines correspond to points featuring the correct relic density of DM. We fixed $\alpha_{D}=0.1$, determined $y_{\mathrm{DM}}$ as described in the text and varied $M_{\chi}$ and $\varepsilon$ using the mass ratios $M_{S}: M_{\chi}: M_{V}=3: 1: 4$ and (a) $\Delta_{\chi}=0.8 M_{\chi}$ or (b) $\Delta_{\chi}=2 M_{\chi}$.

in figure 6 for two values of the splitting parameter $\Delta_{\chi}=0.8 M_{\chi}$ and $\Delta_{\chi}=2 M_{\chi}$. Interestingly, potentially upcoming experiments such as JSNS ${ }^{2}$ [78] or upgraded SeaQuest [20] will have a shorter beam-line and hence can reduce this blind spot. Alternatively, prospective experiments at higher energy, such as FASER [17, 19], could compensate a far detector by a higher boost factor for the heavy state and thus participate in closing the gap. Additionally, the typical relic density prediction is now modified with respect to the standard iDM case and depends strongly on the effective DM coupling with the dark photon and hence on dark Higgs sector properties as shown in eq. (2.9) and eq. (2.10). ${ }^{8}$

A second interesting aspect of this regime occurs when $M_{S}<\Delta_{\chi}$. In this case, the heavy dark sector state $\chi_{2}$ can decay instantaneously by emitting a dark Higgs boson. Given that the dark Higgs boson lifetime is several orders of magnitude larger, the expected reach is drastically reduced. We illustrate this effect in figure 7 .

Secluded regime. The accelerator bounds in this regime are very similar to the iDM case. The main difference is the fact that for a given dark Higgs boson mass, a lower bound on the kinetic mixing parameter is given by the BBN bounds discussed in the previous section. We show this in figure 8 for two typical mass ratios. Note that this bound is weakened in the region of the secluded regime with $M_{\chi} \lesssim M_{S} \lesssim M_{V}$ as the metastable density of dark Higgs boson is depleted by its annihilation into DM. Overall this scenario

${ }^{8}$ For the figure 6 , we have fixed $y_{L}$ and $y_{R}$ such that the effective Yukawa coupling $y_{\mathrm{DM}}$ is given by

$$
y_{D M}=\frac{M_{\chi}}{M_{V}} g_{V} \sqrt{2}\left(1+\frac{\Delta_{\chi}}{4 M_{\chi}}\right),
$$

consistent with the bounds described in appendix A. 


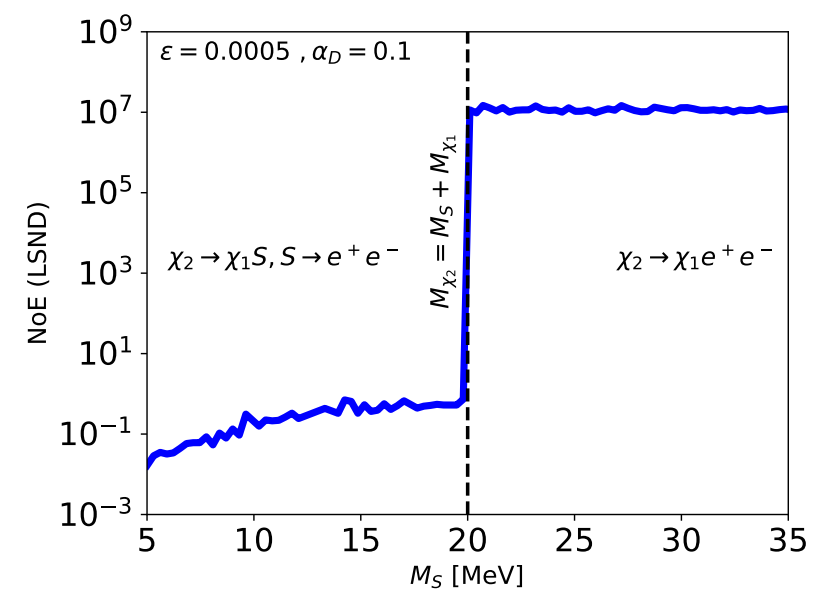

Figure 7. Expected number of events in the LSND experiment for $\varepsilon=5 \times 10^{-4}, \alpha_{D}=0.1, M_{\chi}=$ $40 \mathrm{MeV}$ and $M_{\chi}: M_{V}: \Delta_{\chi}=1: 4: 0.5$ as function of the dark Higgs boson mass $M_{S}$ ( $y_{\mathrm{DM}}$ fixed as described in the text).

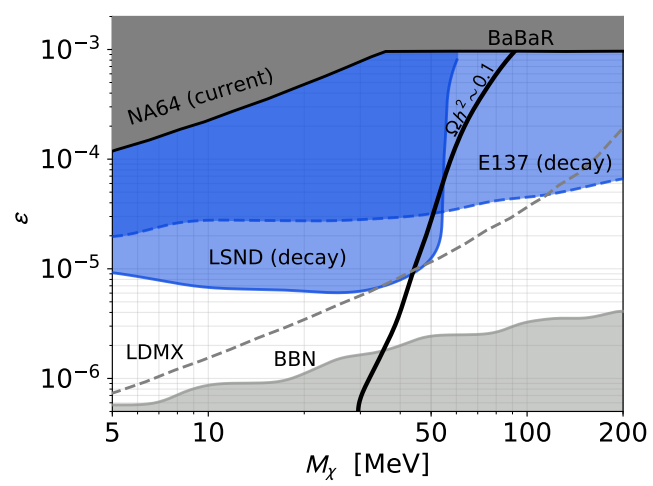

(a)

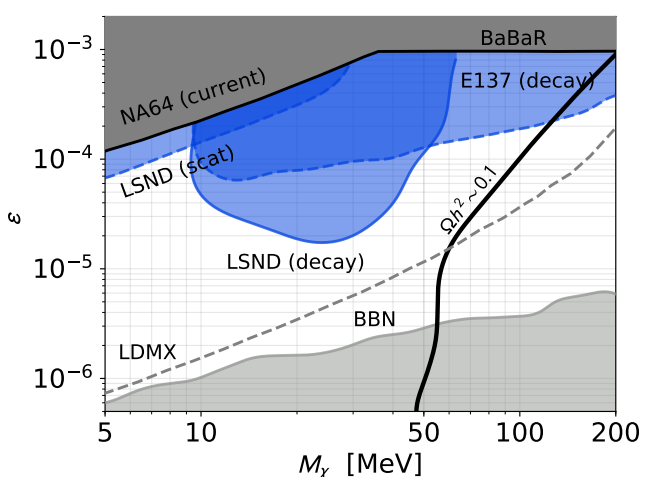

(b)

Figure 8. Bounds for the secluded regime from the E137 [30], LSND [77] arising from DM scattering and $\chi_{2}$ decay (blue regions) and missing energy searches (dark grey regions and grey dashed line for the LDMX projection from [5]). The light grey region at the bottom of the plot are excluded by BBN constraints [6]. The thick black lines correspond to points featuring the correct relic density of DM. We varied $M_{\chi}$ and $\varepsilon$ using $\alpha_{D}=0.8 \times 10^{-3}\left(g_{V}=0.1\right)$ and (a) $M_{S}: M_{\chi}: M_{V}: \Delta_{\chi}=\frac{5}{6}: 1: \frac{5}{2}: 0.3$ or (b) $M_{S}: M_{\chi}: M_{V}: \Delta_{\chi}=\frac{9}{10}: 1: 3: 0.1$. The effective Yukawa coupling between DM of the dark Higgs boson is fixed at 0.005 .

is less constrained than the iDM or mDM ones if we assume that $\chi_{1}$ is the only component of DM, mainly because lower range of $\varepsilon$ are accessible.

Forbidden regime. In this regime, the dark photon cannot decay into DM particles. It is therefore long-lived and typically decays into electrons for the parameter range of interest in this paper. In the absence of any other dark sector states, the typical bounds on the dark photon decay exhibit a mass dependent gap between $10^{-5}-10^{-3}$ (see e.g [5] for 


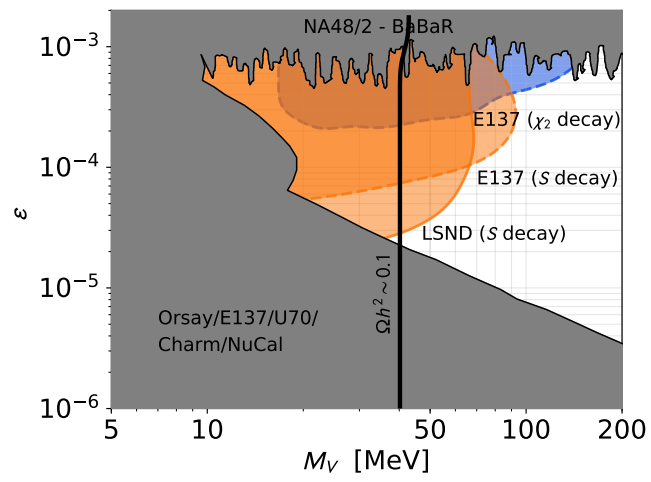

(a)

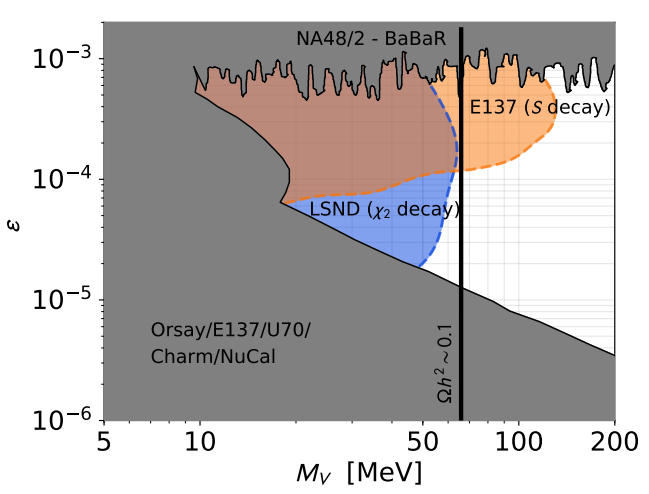

(b)

Figure 9. Bounds on the forbidden regime from the E137 [30], LSND [77] arising from dark Higgs boson decay (orange shaded regions), from visible dark photon searches (dark grey regions from [5]), and from $\chi_{2}$ decay (blue shaded region). The thick black lines correspond to points featuring the correct relic density of DM. We varied $M_{V}$ and $\varepsilon$ using $\alpha_{D}=0.8 \times 10^{-3}\left(g_{V}=0.1\right)$ and (a) $M_{S}: M_{\chi}: M_{V}: \Delta_{\chi}=\frac{5}{3}: 1: \frac{4}{3}: 0.3$ and (b) $M_{S}: M_{\chi}: M_{V}: \Delta_{\chi}=\frac{6}{4}: 1: \frac{5}{4}: 0.75$.

an up-to-date summary). Interestingly, the presence of additional dark sector states can strongly help in closing this blind spot. Indeed, when the dark Higgs boson is heavier than the dark photon, its lifetime is short enough to lead to a sizable number of events in both LSND and E137 experiments. The expected reach then depends on the splitting between the dark Higgs boson and the dark photon which control the dark Higgs boson lifetime (see eq. (3.5)). Furthermore, both the DM and the heavy dark sector state in general can be produced through an off-shell dark photon, albeit at a reduced rate. Depending on the values of the splitting parameters $\Delta_{\chi}$ and $M_{S}-M_{V}$, this can complement or increase the reach from dark Higgs boson decays alone. Overall, dark Higgs boson-related bounds are typically stronger or equivalent to the dark sector decay ones, as we illustrate in figure 9.

It is important to note that these bounds also applies to generic Higgsed dark photon scenario for $M_{S}>M_{V}$ even without the presence of DM.

We have summarised the main features of all four identified regimes in table 1.

\section{Summary and conclusions}

In this paper, we have considered a simple self-consistent model of light (sub-GeV) fermionic DM, whose relic density is fixed by freeze-out. This model is often used when studying pseudo-Dirac DM since large part of its parameter space leads to this paradigm. Our main point is that the same model, when all particles of the spectra are properly accounted for (in particular the dark Higgs boson), leads equally naturally to three other DM regimes: Majorana, secluded and forbidden. The first one corresponds to a simple Majorana DM scenario, albeit with an additional heavy dark sector state $\chi_{2}$, the second relies on DM $t$-channel annihilation into dark Higgs boson, and the last one is based on a thermally suppressed annihilation of DM into dark photons. 


\begin{tabular}{|c|c|c|c|}
\hline Regime & Mass Ranges & $\begin{array}{c}\text { Typical spectrum, } \\
M_{S}: M_{V}: M_{\chi}: \Delta_{\chi}\end{array}$ & Main signatures \\
\hline iDM & $\begin{array}{c}\Delta_{\chi} \ll M_{\chi}, \\
M_{\chi}<M_{S}, M_{V}\end{array}$ & $2: 3: 1: 0.1$ & $\chi_{2} \rightarrow \chi_{1} e^{+} e^{-}$decay, $\chi_{1}$ scattering \\
\hline mDM & $\begin{array}{c}\Delta_{\chi} \sim M_{\chi}, \\
M_{\chi}<M_{S}, M_{V}\end{array}$ & $3: 4: 1: 1$ & $\begin{array}{c}\chi_{2} \rightarrow \chi_{1} e^{+} e^{-} \text {or } \\
\chi_{2} \rightarrow \chi_{1} S, S \rightarrow e^{+} e^{-} \text {decay, } \chi_{1} \\
\text { scattering, presence of a detection } \\
\text { gap for } \varepsilon \sim 10^{-4}\end{array}$ \\
\hline Secluded & $M_{\chi} \gtrsim M_{S}$ & $0.9: 3: 1: 0.1$ & $\begin{array}{c}\chi_{2} \rightarrow \chi_{1} e^{+} e^{-} \text {decay, } \chi_{1} \text { scattering, } \\
\text { BBN bounds on } S\end{array}$ \\
\hline Forbidden & $M_{\chi} \lesssim M_{V} \lesssim M_{S}$ & $\frac{5}{3}: \frac{4}{3}: 1: 0.25$ & $\begin{array}{c}\chi_{2} \rightarrow \chi_{1} e^{+} e^{-}, S \rightarrow V e^{+} e^{-} \text {decay, } \\
\chi_{1} \text { scattering, } V \text { visible decay }\end{array}$ \\
\hline
\end{tabular}

Table 1. Summary of the main features of the four identified regimes, including their typical spectrum with viable dark matter candidate, and main possible signatures from astrophysical observations and in beam-dump experiments.

We have briefly reviewed the cosmological bounds on these scenarios, and then focused on their possible signatures in accelerator-based experiments. The dark Higgs boson has been shown to have an important role in these signatures, either indirectly through its Yukawa couplings to the fermionic states, or directly by either leading to "blind spots" in standard $\chi_{2}$ detection strategies or by its own decay signatures when its lifetime is not too long. We have further considered the current bounds on the four regimes identified stemming from the well studied results of the LSND and E137 experiments. In conclusion, we have shown that similarly to the well known pseudo-Dirac case, the other three scenarios presented here also have bright, albeit distinct prospects in upcoming accelerator-based experiments.

\section{Acknowledgments}

We would like to thank S. Trojanowski for helpful and interesting discussions. LD thanks J. Lesgourgues, F. Kahlhoefer and J. Heisig for interesting discussions regarding CMB bounds. LD, LR and SR are supported in part by the National Science Council (NCN) research grant No. 2015-18-A-ST2-00748. LR is also supported by the project "AstroCeNT: Particle Astrophysics Science and Technology Centre" carried out within the International Research Agendas programme of the Foundation for Polish Science co-financed by the European Union under the European Regional Development Fund. The use of the CIS computer cluster at the National Centre for Nuclear Research is gratefully acknowledged.

\section{A Scan regions and input parameters}

Our model described in section 2.1 has seven free parameters: the kinetic mixing parameter $\varepsilon$, the dark coupling $g_{V}$, the dark Higgs boson mass $\mu_{S}$ and quartic coupling $\lambda_{S}$, the Dirac mass $m_{\chi}$ and the two Higgs boson Yukawa couplings $y_{L}$ and $y_{R}$. Contrary to [6], 


\begin{tabular}{|c|c|c|}
\hline Parameter & Range & Prior \\
\hline$M_{S}$ & $5 \mathrm{MeV}-1 \mathrm{GeV}$ & Log \\
$g_{V}$ & $0.01-2.5$ & Log \\
$M_{V}$ & $10 \mathrm{MeV}-500 \mathrm{MeV}$ & Log \\
$\varepsilon$ & $0.5 \times 10^{-6}-0.001$ & Log \\
$M_{\chi}$ & $-250 \mathrm{MeV}-150 \mathrm{MeV}$ & Linear \\
$\Delta_{\chi}$ & $0.01\left|M_{\chi}\right|-10\left|M_{\chi}\right|$ & Log \\
$y_{D M}$ & $-2-2$ & Linear \\
\hline
\end{tabular}

Table 2. Input parameters for the scans presented in figure 2 .

\begin{tabular}{|l|cccccc|}
\hline Name & Energy & $\pi^{0} / e^{-}$produced & Target Material & Distance & Length & Area \\
\hline LSND & $0.798 \mathrm{GeV}$ & $0.92 \times 10^{23}$ & Water $/$ high-Z metal & $34 \mathrm{~m}$ & $8.3 \mathrm{~m}$ & $25.5 \mathrm{~m}^{2}$ \\
E137 & $20 \mathrm{GeV}$ & $10^{20}$ & $\mathrm{Al}$ & $383 \mathrm{~m}$ & $\sim 1 \mathrm{~m}$ & $8 \mathrm{~m}^{2}$ \\
\hline
\end{tabular}

Table 3. Characteristics of the experiments considered. We define the detector distances from the beam target to the center of the detector. LSND has a cylindrical geometry while E137 has a square intersection with the beam axis. The effective detector distance considered for E137 is developed in the text.

we have traded the five last parameters for more physically relevant ones, namely: the dark Higgs boson mass $M_{S}$ and dark photon mass $M_{V}$, the DM mass $M_{\chi}$, the splitting $\Delta_{\chi} \equiv\left|M_{\chi_{2}}\right|-\left|M_{\chi_{1}}\right|$ between $\chi_{2}$ and $\chi_{1}$, and finally the effective Yukawa between the DM and the dark Higgs boson $y_{\mathrm{DM}}$. We report in table 2, the range used in the scans presented in figure 2. We nonetheless stress that we did not consider Bayesian inference, so that figure 2 should be understood only as an illustration of the four regimes considered.

It is important to note that while this choice of variables makes it particularly easy to relate the physics of DM annihilation to the input variables, the price to pay is that $y_{D M}$ can only belong to a certain range. More precisely, solving directly for $y_{D M}$ as function of the original Lagrangian parameters, we find the accessible range to be:

$$
\frac{M_{\chi}}{M_{V}} g_{V} \sqrt{2}<y_{D M}<\frac{M_{\chi}}{M_{V}} g_{V} \sqrt{2}\left(1+\frac{\Delta_{\chi}}{2 M_{\chi}}\right)
$$

where the sign of the masses are important (in particular, whether or not $M_{\chi}$ is negative). This parameter is of course of importance to both the secluded regime, for which we typically consider $M_{\chi}<0$ and $y_{D M} \ll 1$, which satisfies eq. (A.1), and the Majorana case, for which typically $M_{\chi}>0$ and the bounds on $y_{D M}$ are of importance.

\section{B Monte-Carlo setup and numerical simulations}

We present in this appendix the numerical setup used to obtain the number of events in the experiments E137 and LSND, whose main characteristics are described in table 3. 


\section{B.1 Production at electron and proton beam dump}

In proton beam dump experiment (and more particularly LSND), the dominant production mechanism for a beam energy in the tens of $\mathrm{GeV}$ is the decay of light mesons. In this paper, we build upon the numerical tools developed in [6] for this setup and based on a modified version of the code BdNMC [26]. Focusing on LSND, we start by simulating the kinematic distribution of the light meson $\pi^{0}$ based on a weighted Burman-Smith distribution in order to account for the various target material (water, then high-Z metal) used over the experiment lifetime. The (differential) branching ratios for $\pi^{0}$ decay into the relevant final states $\chi_{i} \chi_{j} \gamma$ and $S V \gamma$ are then calculated analytically and used to sample the kinematics of the final state and the total number of produced events. More details can be found in [6], including the expression for the differential decay rate for $\pi^{0} \rightarrow S V \gamma$, while the rate for $\pi^{0} \rightarrow \chi_{i} \chi_{j} \gamma$ is shown in eq. (3.2). When the heavy dark sector state's $\left(\chi_{2}\right)$ fast decay into $S \chi_{1}$ is kinematically available, we decay it by assuming the process to occur instantaneously. Depending on the final search channel we are interested in (dark matter scattering, $\chi_{2}$ decay or $S$ decay) the relevant particle kinematics are then stored and passed to the detector simulation part of the code.

We proceed differently for electron beam dump in that we instead rely on the public code Madgraph [76] to produce the events. In our calculation, we use the following form factor for the target atom with an elastic and inelastic term given by [31, 79-81]

$$
\begin{aligned}
G_{2}^{\mathrm{el}} & =\left(\frac{a^{2} t}{1+a^{2} t}\right)^{2}\left(\frac{1}{1+t / d}\right)^{2} Z^{2} \\
G_{2}^{\mathrm{in}} & =\left(\frac{a^{\prime 2} t}{1+a^{\prime 2} t}\right)^{2}\left(\frac{1+\frac{t}{4 m_{p}^{2}}\left(\mu_{p}^{2}-1\right)}{\left(1+\frac{t}{0.71 \mathrm{GeV}^{2}}\right)^{4}}\right)^{2} Z
\end{aligned}
$$

where $a=111 Z^{-1 / 3} / m_{e}$ with $m_{e}$ being the electron mass, $d=0.164 \mathrm{GeV}^{2} A^{-2 / 3}$ with $A$ being the number of nucleons in the target, $a^{\prime}=773 Z^{-2 / 3} / m_{e}$ with $Z$ being the number of protons in the target, $m_{p}$ is the proton mass and $\mu_{p}=2.79$. The atom-dark photon vertex then becomes $\left(P_{i}+P_{f}\right)_{\mu} \sqrt{G_{2}}$, where $P_{i}$ and $P_{f}$ are initial and final momenta of the atom respectively, while $G_{2}=G_{2}^{\text {el }}+G_{2}^{\text {in }}$. We implement the above atomic and nuclear form factors using the in-built procedure of Madgraph [82].

In order to account for the energy damping of the electron beam in E137 as it goes through the aluminum target, we run the previous code for three different energies of $18 \mathrm{GeV}, 10.5 \mathrm{GeV}$ and $3.5 \mathrm{GeV}$. The resulting cross-section is then weighted by the usual energy distribution from ref. [81]:

$$
I\left(E_{0}, E_{e}\right)=\frac{E_{0}}{E_{e}} \int_{0}^{\infty} d s \frac{1}{E_{o}} \frac{\left[\ln \left(E_{0} / E_{e}\right)\right]^{4 s / 3-1}}{\Gamma[4 s / 3]},
$$

where we have directly integrated over the thick target length(approximated to infinity), 
$E_{0}=20 \mathrm{GeV}$ and $E_{e}=18,10.5,3.5 \mathrm{GeV}$ in our case. ${ }^{9}$ Finally we reconstruct an unweighted sample of final states by adding events from our three energy bins proportionally to the bin's weight. This unweighted sample of final states' kinematic is then passed to the detector simulation part of the code, along with the total number of produced events.

\section{B.2 Decay and detector simulations}

This part of our simulation, also loosely based on BdNMC [26], takes as input the kinematics information of final states and the total number of produced events (regardless of the electron or proton beam dump origin of the events). The scattering detection simulation is imported from the original BdNMC code and is described in [26]. In this section, we will focus on the detector simulation for a decaying $\chi_{2}$ or $S$ particles.

We start by analytically calculating the lifetime of the particle and its decay probability within a given decay volume consisting of the detector itself for LSND and a fraction of the open space before the detector for E137 (we will comment on this particular aspect at the end of this section). We then sample the decay product kinematics using the hardcoded expression for the differential decay rate for the processes $\chi_{2} \rightarrow \chi_{1} e^{+} e^{-}, S \rightarrow e^{+} e^{-}$ or $S \rightarrow V e^{+} e^{-}$. At this point we determine whether any more decay occurs before the detector (e.g dark photon decay for the $S \rightarrow V e^{+} e^{-}$case) then pass the decay products to the detector simulation.

First, the simulation of the LSND detector response to the event is based on the search in [83], so that we require the electrons in final state to either be reconstructed as a single track (namely with an angular spread of the $e^{+} e^{-}$pair below $12^{\circ}$ ) or that only one of the electron is reconstructed (using an electron detection efficiency of 19\%). Overall, the reconstructed object is then required to have an energy between 18 and $50 \mathrm{MeV}$ and be forward-oriented with $\cos \theta_{b}>0.9$, where $\theta_{b}$ is the angle with the beam-line in the laboratory frame, in order to fake the signature of an elastic events as searched for in [83]. Following [73], we then use a 55-events limit to derive the bounds from this experiment.

Second, for the E137 experiment, we require that at least one of the electrons crosses the detector with an angle to the beam line smaller than $30 \mathrm{mrad}$ and an energy $E>1 \mathrm{GeV} .{ }^{10}$ No such events were observed by E137 (see, e.g figure 9 of ref. [30]) so that we place an upper limit of 3-events. An important comment regarding E137 is that the decay volume in front of the detector was an open space at atmospheric pressure. The typical radiation length for electrons in the air is of $304 \mathrm{~m}$ [84], but perhaps more importantly the corresponding Moliere radius is $73 \mathrm{~m}$. This implies that after a distance of order meter, the energy of the electromagnetic shower is spread out in a transverse direction significantly larger than the spread of an event as seen by the E137 (see figure 8 of ref. [30]). While a proper modeling

\footnotetext{
${ }^{9}$ Importantly, when accounting for the energy damping, we did not include the transverse broadening of the beam as it looses energy in the target. This should further reduce the number of events in the low energy bin. However, the high energy threshold of the E137 experiment implies that most of the events from this bin do not eventually pass the detector cuts, so that including this effect should not modify our result significantly.

${ }^{10}$ Note that the precise value of the threshold energy to be used for this experiment is somewhat controversial in the literature [20].
} 
of the electromagnetic shower will be probably needed to go beyond the order of magnitude evaluation, we will take the simpler approach of considering only those events for which the decay occurs within $\sim 1 \mathrm{~m}$ of the detector.

Open Access. This article is distributed under the terms of the Creative Commons Attribution License (CC-BY 4.0), which permits any use, distribution and reproduction in any medium, provided the original author(s) and source are credited.

\section{References}

[1] L. Roszkowski, E.M. Sessolo and S. Trojanowski, WIMP dark matter candidates and searches - current status and future prospects, Rept. Prog. Phys. 81 (2018) 066201 [arXiv: 1707.06277] [INSPIRE].

[2] T. Plehn, Yet Another Introduction to Dark Matter, arXiv:1705.01987 [INSPIRE].

[3] G. Arcadi et al., The waning of the WIMP? A review of models, searches and constraints, Eur. Phys. J. C 78 (2018) 203 [arXiv:1703.07364] [inSPIRE].

[4] J. Alexander et al., Dark Sectors 2016 Workshop: Community Report, FERMILAB-CONF-16-421 [arXiv: 1608.08632] [INSPIRE].

[5] M. Battaglieri et al., US Cosmic Visions: New Ideas in Dark Matter 2017: Community Report, FERMILAB-CONF-17-282 [arXiv: 1707.04591] [INSPIRE].

[6] L. Darmé, S. Rao and L. Roszkowski, Light dark Higgs boson in minimal sub-GeV dark matter scenarios, JHEP 03 (2018) 084 [arXiv:1710.08430] [INSPIRE].

[7] D. Tucker-Smith and N. Weiner, Inelastic dark matter, Phys. Rev. D 64 (2001) 043502 [hep-ph/0101138] [INSPIRE].

[8] M. Pospelov, A. Ritz and M.B. Voloshin, Secluded WIMP Dark Matter, Phys. Lett. B 662 (2008) 53 [arXiv: 0711.4866] [INSPIRE].

[9] M. Pospelov, Secluded U(1) below the weak scale, Phys. Rev. D 80 (2009) 095002 [arXiv:0811.1030] [INSPIRE].

[10] B. Batell, M. Pospelov and A. Ritz, Probing a Secluded U(1) at B-factories, Phys. Rev. D 79 (2009) 115008 [arXiv:0903.0363] [inSPIRE].

[11] N. Arkani-Hamed, D.P. Finkbeiner, T.R. Slatyer and N. Weiner, A Theory of Dark Matter, Phys. Rev. D 79 (2009) 015014 [arXiv:0810.0713] [inSPIRE].

[12] M. Duerr, A. Grohsjean, F. Kahlhoefer, B. Penning, K. Schmidt-Hoberg and C. Schwanenberger, Hunting the dark Higgs, JHEP 04 (2017) 143 [arXiv:1701.08780] [INSPIRE].

[13] A. Ahmed, M. Duch, B. Grzadkowski and M. Iglicki, Multi-Component Dark Matter: the vector and fermion case, Eur. Phys. J. C 78 (2018) 905 [arXiv:1710. 01853] [INSPIRE].

[14] F.C. Correia and S. Fajfer, Restrained dark U(1) d at low energies, Phys. Rev. D 94 (2016) 115023 [arXiv: 1609.00860] [INSPIRE].

[15] S. Knapen, T. Lin and K.M. Zurek, Light Dark Matter: Models and Constraints, Phys. Rev. D 96 (2017) 115021 [arXiv: 1709.07882] [INSPIRE].

[16] J.L. Feng and J. Smolinsky, Impact of a resonance on thermal targets for invisible dark photon searches, Phys. Rev. D 96 (2017) 095022 [arXiv:1707.03835] [INSPIRE]. 
[17] J.L. Feng, I. Galon, F. Kling and S. Trojanowski, Dark Higgs bosons at the ForwArd Search ExpeRiment, Phys. Rev. D 97 (2018) 055034 [arXiv:1710.09387] [InSPIRE].

[18] B. Wojtsekhowski et al., Searching for a dark photon: Project of the experiment at VEPP-3, 2018 JINST 13 P02021 [arXiv:1708.07901] [INSPIRE].

[19] J.L. Feng, I. Galon, F. Kling and S. Trojanowski, ForwArd Search ExpeRiment at the LHC, Phys. Rev. D 97 (2018) 035001 [arXiv: 1708.09389] [InSPIRE].

[20] A. Berlin, S. Gori, P. Schuster and N. Toro, Dark Sectors at the Fermilab SeaQuest Experiment, Phys. Rev. D 98 (2018) 035011 [arXiv:1804.00661] [InSPIRE].

[21] A. Berlin, N. Blinov, G. Krnjaic, P. Schuster and N. Toro, Dark Matter, Millicharges, Axion and Scalar Particles, Gauge Bosons and Other New Physics with LDMX, arXiv:1807.01730 [INSPIRE].

[22] J.R. Jordan, Y. Kahn, G. Krnjaic, M. Moschella and J. Spitz, Signatures of Pseudo-Dirac Dark Matter at High-Intensity Neutrino Experiments, Phys. Rev. D 98 (2018) 075020 [arXiv: 1806.05185] [INSPIRE].

[23] B. Batell, M. Pospelov and A. Ritz, Exploring Portals to a Hidden Sector Through Fixed Targets, Phys. Rev. D 80 (2009) 095024 [arXiv: 0906.5614] [InSPIRE].

[24] B. Batell, R. Essig and Z. Surujon, Strong Constraints on Sub-GeV Dark Sectors from SLAC Beam Dump E137, Phys. Rev. Lett. 113 (2014) 171802 [arXiv:1406.2698] [inSPIRE].

[25] E. Izaguirre, G. Krnjaic, P. Schuster and N. Toro, Analyzing the Discovery Potential for Light Dark Matter, Phys. Rev. Lett. 115 (2015) 251301 [arXiv:1505.00011] [INSPIRE].

[26] P. deNiverville, C.-Y. Chen, M. Pospelov and A. Ritz, Light dark matter in neutrino beams: production modelling and scattering signatures at MiniBooNE, T2K and SHiP, Phys. Rev. D 95 (2017) 035006 [arXiv: 1609.01770] [INSPIRE].

[27] BDX collaboration, M. Battaglieri et al., Dark Matter Search in a Beam-Dump eXperiment (BDX) at Jefferson Lab, arXiv:1607.01390 [INSPIRE].

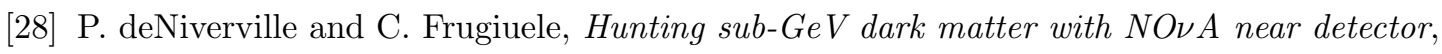
arXiv: 1807.06501 [INSPIRE].

[29] LSND collaboration, C. Athanassopoulos et al., The liquid scintillator neutrino detector and LAMPF neutrino source, Nucl. Instrum. Meth. A 388 (1997) 149 [nucl-ex/9605002] [INSPIRE].

[30] J.D. Bjorken et al., Search for Neutral Metastable Penetrating Particles Produced in the SLAC Beam Dump, Phys. Rev. D 38 (1988) 3375 [inSPIRE].

[31] J.D. Bjorken, R. Essig, P. Schuster and N. Toro, New Fixed-Target Experiments to Search for Dark Gauge Forces, Phys. Rev. D 80 (2009) 075018 [arXiv:0906. 0580] [INSPIRE].

[32] P. Schuster, N. Toro and I. Yavin, Terrestrial and Solar Limits on Long-Lived Particles in a Dark Sector, Phys. Rev. D 81 (2010) 016002 [arXiv:0910.1602] [INSPIRE].

[33] R. Essig, P. Schuster and N. Toro, Probing Dark Forces and Light Hidden Sectors at Low-Energy $e^{+} e^{-}$Colliders, Phys. Rev. D 80 (2009) 015003 [arXiv:0903.3941] [InSPIRE].

[34] J. Ellis, M. Fairbairn and P. Tunney, Anomaly-Free Dark Matter Models are not so Simple, JHEP 08 (2017) 053 [arXiv: 1704.03850] [INSPIRE].

[35] B. Holdom, Two U(1)'s and Epsilon Charge Shifts, Phys. Lett. B 166 (1986) 196 [InSPIRE]. 
[36] K.S. Babu, C.F. Kolda and J. March-Russell, Implications of generalized Z-Z' mixing, Phys. Rev. D 57 (1998) 6788 [hep-ph/9710441] [INSPIRE].

[37] D.E. Morrissey and A.P. Spray, New Limits on Light Hidden Sectors from Fixed-Target Experiments, JHEP 06 (2014) 083 [arXiv:1402.4817] [INSPIRE].

[38] W. Porod, SPheno, a program for calculating supersymmetric spectra, SUSY particle decays and SUSY particle production at $e^{+} e^{-}$colliders, Comput. Phys. Commun. 153 (2003) 275 [hep-ph/0301101] [INSPIRE].

[39] W. Porod and F. Staub, SPheno 3.1: Extensions including flavour, CP-phases and models beyond the MSSM, Comput. Phys. Commun. 183 (2012) 2458 [arXiv:1104.1573] [inSPIRE].

[40] F. Staub, SARAH, arXiv:0806.0538 [INSPIRE].

[41] F. Staub, SARAH 3.2: Dirac Gauginos, UFO output and more, Comput. Phys. Commun. 184 (2013) 1792 [arXiv:1207.0906] [INSPIRE].

[42] F. Staub, SARAH 4: A tool for (not only SUSY) model builders, Comput. Phys. Commun. 185 (2014) 1773 [arXiv:1309.7223] [INSPIRE].

[43] F. Feroz, M.P. Hobson and M. Bridges, MultiNest: an efficient and robust Bayesian inference tool for cosmology and particle physics, Mon. Not. Roy. Astron. Soc. 398 (2009) 1601 [arXiv: 0809.3437] [INSPIRE].

[44] Planck collaboration, P.A.R. Ade et al., Planck 2015 results. XIII. Cosmological parameters, Astron. Astrophys. 594 (2016) A13 [arXiv:1502.01589] [INSPIRE].

[45] G. Bélanger, F. Boudjema, A. Pukhov and A. Semenov, MicrOMEGAs4.1: two dark matter candidates, Comput. Phys. Commun. 192 (2015) 322 [arXiv:1407.6129] [INSPIRE].

[46] T.R. Slatyer, Indirect dark matter signatures in the cosmic dark ages. I. Generalizing the bound on s-wave dark matter annihilation from Planck results, Phys. Rev. D 93 (2016) 023527 [arXiv: 1506.03811] [INSPIRE].

[47] M. Duerr, K. Schmidt-Hoberg and S. Wild, Self-interacting dark matter with a stable vector mediator, JCAP 09 (2018) 033 [arXiv:1804.10385] [INSPIRE].

[48] R.T. D'Agnolo, C. Mondino, J.T. Ruderman and P.-J. Wang, Exponentially Light Dark Matter from Coannihilation, JHEP 08 (2018) 079 [arXiv: 1803.02901] [INSPIRE].

[49] R.T. D'Agnolo and J.T. Ruderman, Light Dark Matter from Forbidden Channels, Phys. Rev. Lett. 115 (2015) 061301 [arXiv: 1505.07107] [INSPIRE].

[50] A. Fradette and M. Pospelov, BBN for the LHC: constraints on lifetimes of the Higgs portal scalars, Phys. Rev. D 96 (2017) 075033 [arXiv:1706.01920] [InSPIRE].

[51] T.R. Slatyer and C.-L. Wu, General Constraints on Dark Matter Decay from the Cosmic Microwave Background, Phys. Rev. D 95 (2017) 023010 [arXiv: 1610.06933] [INSPIRE].

[52] K.M. Nollett and G. Steigman, BBN And The CMB Constrain Light, Electromagnetically Coupled WIMPs, Phys. Rev. D 89 (2014) 083508 [arXiv:1312.5725] [INSPIRE].

[53] A. Burrows and J.M. Lattimer, The birth of neutron stars, Astrophys. J. 307 (1986) 178 [INSPIRE].

[54] A. Burrows and J.M. Lattimer, Neutrinos from SN 1987A, Astrophys. J. 318 (1987) L63 [INSPIRE]. 
[55] G. Raffelt and D. Seckel, Bounds on Exotic Particle Interactions from SN 1987a, Phys. Rev. Lett. 60 (1988) 1793 [INSPIRE].

[56] G.G. Raffelt, Stars as laboratories for fundamental physics, Chicago University Press, Chicago, U.S.A., (1996).

[57] J.B. Dent, F. Ferrer and L.M. Krauss, Constraints on Light Hidden Sector Gauge Bosons from Supernova Cooling, arXiv:1201.2683 [INSPIRE].

[58] H. An, M. Pospelov and J. Pradler, New stellar constraints on dark photons, Phys. Lett. B 725 (2013) 190 [arXiv: 1302.3884] [INSPIRE].

[59] J. Redondo and G. Raffelt, Solar constraints on hidden photons re-visited, JCAP 08 (2013) 034 [arXiv: 1305.2920] [inSPIRE].

[60] D. Kazanas, R.N. Mohapatra, S. Nussinov, V.L. Teplitz and Y. Zhang, Supernova Bounds on the Dark Photon Using its Electromagnetic Decay, Nucl. Phys. B 890 (2014) 17 [arXiv: 1410.0221] [INSPIRE].

[61] E. Rrapaj and S. Reddy, Nucleon-nucleon bremsstrahlung of dark gauge bosons and revised supernova constraints, Phys. Rev. C 94 (2016) 045805 [arXiv: 1511.09136] [INSPIRE].

[62] J.H. Chang, R. Essig and S.D. McDermott, Revisiting Supernova 1987A Constraints on Dark Photons, JHEP 01 (2017) 107 [arXiv: 1611.03864] [INSPIRE].

[63] E. Hardy and R. Lasenby, Stellar cooling bounds on new light particles: plasma mixing effects, JHEP 02 (2017) 033 [arXiv: 1611.05852] [INSPIRE].

[64] C. Mahoney, A.K. Leibovich and A.R. Zentner, Updated Constraints on Self-Interacting Dark Matter from Supernova 1987A, Phys. Rev. D 96 (2017) 043018 [arXiv:1706.08871] [INSPIRE].

[65] J.H. Chang, R. Essig and S.D. McDermott, Supernova 1987A Constraints on Sub-GeV Dark Sectors, Millicharged Particles, the QCD Axion and an Axion-like Particle, JHEP 09 (2018) 051 [arXiv: 1803.00993] [inSPIRE].

[66] S. Andreas et al., Proposal for an Experiment to Search for Light Dark Matter at the SPS, arXiv:1312. 3309 [INSPIRE].

[67] E. Izaguirre, G. Krnjaic, P. Schuster and N. Toro, Testing GeV-Scale Dark Matter with Fixed-Target Missing Momentum Experiments, Phys. Rev. D 91 (2015) 094026 [arXiv: 1411.1404] [INSPIRE].

[68] BaBAR collaboration, J.P. Lees et al., Search for Invisible Decays of a Dark Photon Produced in $e^{+} e^{-}$Collisions at BaBar, Phys. Rev. Lett. 119 (2017) 131804 [arXiv: 1702.03327] [INSPIRE].

[69] NA64 collaboration, D. Banerjee et al., Search for vector mediator of Dark Matter production in invisible decay mode, Phys. Rev. D 97 (2018) 072002 [arXiv:1710.00971] [INSPIRE].

[70] NA48/2 collaboration, J.R. Batley et al., Search for the dark photon in $\pi^{0}$ decays, Phys. Lett. B 746 (2015) 178 [arXiv: 1504.00607] [INSPIRE].

[71] BABAR collaboration, J.P. Lees et al., Search for a Dark Photon in $e^{+} e^{-}$Collisions at BaBar, Phys. Rev. Lett. 113 (2014) 201801 [arXiv: 1406. 2980] [InSPIRE].

[72] LHCb collaboration, Search for Dark Photons Produced in 13 TeV pp Collisions, Phys. Rev. Lett. 120 (2018) 061801 [arXiv: 1710.02867] [INSPIRE]. 
[73] E. Izaguirre, Y. Kahn, G. Krnjaic and M. Moschella, Testing Light Dark Matter Coannihilation With Fixed-Target Experiments, Phys. Rev. D 96 (2017) 055007 [arXiv: 1703.06881] [INSPIRE].

[74] MiniBoonE DM collaboration, A.A. Aguilar-Arevalo et al., Dark Matter Search in Nucleon, Pion and Electron Channels from a Proton Beam Dump with MiniBooNE, arXiv: 1807.06137 [INSPIRE].

[75] L. Darmé, S. Rao and L. Roszkowski, Light dark sector at colliders and fixed target experiments, in 53rd Rencontres de Moriond on QCD and High Energy Interactions (Moriond QCD 2018) La Thuile, Italy, March 17-24, 2018, arXiv:1805.06179 [INSPIRE].

[76] J. Alwall et al., The automated computation of tree-level and next-to-leading order differential cross sections and their matching to parton shower simulations, JHEP 07 (2014) 079 [arXiv: 1405.0301] [INSPIRE].

[77] LSND collaboration, L.B. Auerbach et al., Measurement of electron - neutrino - electron elastic scattering, Phys. Rev. D 63 (2001) 112001 [hep-ex/0101039] [INSPIRE].

[78] S. Ajimura et al., Technical Design Report (TDR): Searching for a Sterile Neutrino at J-PARC MLF (E56, JSNS2), arXiv:1705.08629 [INSPIRE].

[79] K.J. Kim and Y.-S. Tsai, Improved Weizsäcker-Williams method and its application to lepton and W-boson pair production, Phys. Rev. D 8 (1973) 3109 [INSPIRE].

[80] Y.-S. Tsai, Pair Production and Bremsstrahlung of Charged Leptons, Rev. Mod. Phys. 46 (1974) 815 [Erratum ibid. 49 (1977) 521] [INSPIRE].

[81] Y.-S. Tsai, Axion Bremsstrahlung by an electron beam, Phys. Rev. D 34 (1986) 1326 [INSPIRE].

[82] https://cp3.irmp.ucl.ac.be/projects/madgraph/wiki/FormFactors.

[83] LSND collaboration, A. Aguilar-Arevalo et al., Evidence for neutrino oscillations from the observation of $\bar{\nu}_{e}$ appearance in a $\bar{\nu}_{\mu}$ beam, Phys. Rev. D 64 (2001) 112007 [hep-ex/0104049] [INSPIRE].

[84] Particle Data Group collaboration, C. Patrignani et al., Review of Particle Physics, Chin. Phys. C 40 (2016) 100001 [inSPIRE]. 